\title{
A NOTE ON THE SECOND SMALLEST PRIME $k$ TH POWER NONRESIDUE
}

\author{
RICHARD H. HUDSON
}

\begin{abstract}
Upper bounds for the second smallest prime $k$ th power nonresidue, which we denote by $g_{2}(p, k)$, have been given by many authors. Theorem 1 represents an improvement of these bounds, at least for odd $k$. We also give specific estimates for $g_{2}(p, k)$, and an upper bound for the $n$th $(n \geq 2)$ smallest prime $k$ th power nonresidue as a function of the first $n-1$ prime nonresidues. Upper bounds for $g_{2}(p, k)$ should take on new interest since the author has shown elsewhere that the first two consecutive $k$ th power nonresidues are bounded above by the product of the first two prime nonresidues.
\end{abstract}

1. Introduction. Throughout $k$ will be an integer $\geq 2$ and $p$ will be a prime $\equiv 1(\bmod k)$. The $n$th smallest prime kth power nonresidue, $n \geq 2$, will be denoted by $g_{n}(p, k)$. In [4] the author investigated at some length the problem of finding upper bounds for $g_{2}(p, k)$. The major purpose of this paper is to improve the bounds given in [4, Theorem 4$]$, which are, to the best of our knowledge, the sharpest upper bounds known for $g_{2}(p, k)$ for odd $k$. In particular, we are now able to prove the following result.

Theorem 1. For each $\epsilon>0$ and $p \geq 5$,

$$
g_{2}(p, k)=\mathcal{O}_{\epsilon, k}\left(p^{k / 4(k-1)+\epsilon}\right) \text {. }
$$

We also note that several other theorems in [4] can be improved using recent work of P. D. T. A. Elliott [2], [3], Hugh L. Montgomery [8], and K. K. Norton [9], [10].

In the following proof, $h_{j}(p, k), j=0,1, \ldots, k-1$, will denote the smallest positive representative of the $j$ th coset formed with respect to the subgroup of the $k$ th powers $\bmod p$. In particular, for all $k, h_{0}(p, k)=1, h_{1}(p, k)$ $=g_{1}(p, k), h_{2}(p, k)$ denotes the smallest positive $k$ th power nonresidue in a coset different than the coset to which $h_{1}(p, k)$ belongs, $h_{k-1}$ denotes the

Received by the editors October 24, 1973.

AMS (MOS) subject classifications (1970). Primary 10A15, $10 \mathrm{H} 35$. 
smallest positive $k$ th power nonresidue in a coset different than the cosets to which $h_{1}, h_{2}, \ldots, h_{k-2}$ belong. In the following proof we assume that $k \geq 3$ since Theorem 1 is well known for $k=2$. We also observe that $g_{2}(p, k)$ is less than $p$ if $p \geq 5$, since otherwise the $k$ th power nonresidues of $p$ consist only of powers of $g_{1}(p, k)$ and these are clearly insufficiently numerous.

2. Proof of Theorem 1. We want to show that for each $\epsilon>0$ and $k \geq 3$ there exists a constant $c_{1}(\epsilon, k)$ such that for every prime $p \geq 5$,

$$
g_{2}(p, k)<c_{1}(\epsilon, k) p^{k / 4(k-1)+\epsilon} .
$$

Assume first, that for each $\epsilon>0$ and $k \geq 3$, there exists a constant $c_{2}(\epsilon, k)$ such that for every odd prime $p$,

$$
g_{1}(p, k)<c_{2}(\epsilon, k) p^{1 / 4(k-1)+\epsilon /(k-1)} .
$$

It follows from [4, Lemma 2] that

$$
g_{2}(p, k) \leq g_{1}(p, k) \cdot s_{n}+1
$$

where $s_{n}$ denotes the maximum number of consecutive integers in any of the nonresidue cosets formed with respect to the subgroup of $k$ th powers mod $p$. It is well known that $s_{n}<c_{3} p^{1 / 4} \log p$ for all $k$ where $c_{3}$ is an absolute constant (in fact $c_{3}<3.230$; see [6]). Of course, $\log p=o\left(p^{\epsilon /(k-1)}\right)$ and, consequently, if (2.2) holds, (2.1) follows at once from (2.3).

Conversely, assume there exists $\epsilon>0$ or $k \geq 3$ such that for every constant $c_{4}(\epsilon, k)$, there exist infinitely many primes with

$$
g_{1}(p, k)>c_{4}(\epsilon, k) p^{1 / 4(k-1)+\epsilon /(k-1)} .
$$

Norton [9] has shown that for each $\epsilon>0$ and $k \geq 2$ there must exist a constant $c_{\varsigma}(\epsilon, k)$ such that for every odd prime $p$,

$$
b_{k-1}(p, k)<c_{5}(\epsilon, k) p^{1 / 4+\epsilon} .
$$

If (2.4) and (2.5) both hold, we must have $h_{k-1}(p, k)<\left(g_{1}(p, k)\right)^{k-1}$.

But if $g_{2}(p, k)>h_{k-1}(p, k)$, and if $x$ is any $k$ th power nonresidue such that $1<x \leq h_{k-1}(p, k)$, then clearly $x=\left(g_{1}(p, k)\right)^{a} y$, where $y$ is a $k$ th power residue and $1 \leq a \leq k-2$. Hence, the inequalities (2.4) and $g_{2}(p, k)$ $>h_{k-1}(p, k)$ imply that there are at most $k-1$ cosets of the subgroup of $k$ th powers $\bmod p$, a contradiction. Consequently, if (2.4) holds, we have $g_{2}(p, k) \leq h_{k-1}(p, k)$, and (2.1) follows from (2.5). 
3. Specific estimates. We shall call an upper bound for $g_{n}(p, k)$ a specific estimate if it is of the form $g_{n}(p, k)<c p^{\alpha}$, where $c$ and $\alpha$ are specified real numbers and the bound holds for all $p$ greater than a specified real number. We shall call a specific estimate a universal specific estimate if it is a specific estimate which holds for all $p$ for which $g_{n}(p, k)$ exists.

L. K. Hua [7] has given the best specific estimate for $g_{2}(p, k)$ for $k=$ 2. In particular Hua showed that for $k=2$ (and hence for even $k$ ) and $p>$ $e^{250}$

$$
g_{2}(p, k)<(57600 p)^{5 / 16} \text {. }
$$

Using [4, Theorem 3] and K. K. Norton's [10] recently announced improvement of his universal specific estimate for $g_{1}(p, k)$, namely $g_{1}(p, k)<$ $1.1 p^{1 / 4}(\log p+4)$, it is possible to slightly improve Corollary 1 in $[4, \mathrm{p} .103]$.

Theorem 2. For each $k$ and all $p \geq 5$,

$$
g_{2}(p, k)<4 p^{7 / 16}(1.1 \log p+4.4)^{3 / 4}+8.8 p^{1 / 4} \log p+36.2
$$

Norton [10] has also announced a universal specific estimate for the maximum number, $S$, of consecutive integers in any coset formed with respect to the subgroup of $k$ th powers $\bmod p$, namely $S<4.1 p^{1 / 4} \log p$. The author has shown in [6] that this estimate can be improved to $S<3.616 p^{1 / 4} \log p$. This allows us to make specific our estimate [4, Lemma 3] for the $n$th smallest prime $k$ th power nonresidue as a function of the first $n-1$ prime nonresidues.

Theorem 3. Let $n$ be any integer $\geq 2$. Then

$$
g_{n}(p, k)<\left(3.616 p^{1 / 4} \log p+1\right)\left(\prod_{r=1}^{n-1} g_{r}(p, k)\right)+1 .
$$

In [5] the author noted that if $g_{1}(p, k)<2^{1 / 2} p^{1 / 4}$, then $S<2.9086 p^{1 / 4} \log p$. This yields the following exemplary corollary to Theorem 3 .

Corollary. Let $p$ be a prime for which $g_{1}(p, k)=2$ so that $g_{2}(p, k)$ is the smallest odd kth power nonresidue. Then

$$
g_{2}(p, k)<5.8172 p^{1 / 4} \log p+3 \text {. }
$$

This universal specific estimate for the smallest odd $k$ th power nonresidue improves earlier estimates of Brauer [1] and the author [4, Theorem 1]. In conclusion, we note that Theorem 7 of [4] appears rather naive in 
retrospect. In fact Hugh L. Montgomery has informed me that if the generalized Riemann hypothesis is true, then $g_{n}(p, k)=\mathcal{O}\left(\log ^{2} p\right)$ for all $n<$ $\log ^{2} p / \log \log p$; see also [6].

Note added in Proof (July, 1974). In the near future we hope to improve Theorem 1 considerably. In particular we expect to prove, without hypotheses, that $g_{n}(p, k)=\mathcal{O}_{\epsilon}\left(p^{1 / 4+\epsilon}\right)$ for every $p>p_{0}(\epsilon)$ and every $n \leq(c \log p) / \log \log p$ (for some positive constant $c$ ).

\section{REFERENCES}

1. A. Brauer, On the non-existence of the Euclidean algorithm in certain quadratic number fields, Amer. J. Math. 62 (1940), 697-716. MR 2, 146.

2. P. D. T. A. Elliott, On the mean value of $f(p)$, Proc. London Math. Soc. (3) 21 (1970), 28-96. MR $42 \# 1783$.

3. - On the least pair of consecutive quadratic non-residues ( $\bmod p)$, Proc. Number Theory Conference, Univ. Colorado, Boulder, Colo., 1972, pp. 75-79.

4. Richard H. Hudson, Prime kth power non-residues, Acta Arith. 23 (1973), 89-106.

5. - A bound for the first occurrence of three consecutive integers with equal quadratic character, Duke Math. J. 40 (1973), 33-39.

6. - The least pair of consecutive kth power non-residues (to appear).

7. L. K. Hua, $O_{n}$ the distribution of quadratic non-residues and the Euclidean algorithm in real quadratic fields. I, Trans. Amer. Math. Soc. 56 (1944), 537-546. MR 6, 170.

8. Hugh L. Montgomery, Topics in multiplicative number theory, Lecture Notes in Math., vol. 227, Springer-Verlag, Berlin, 1971.

9. K. K. Norton, Upper bounds for kth power coset representatives modulo n, Acta Arith. 15 (1968/69), 161-179. MR 39 \# 1419.

10. —_, Bounds for sequences of consecutive power residues. I, Proc. Sympos. Pure Math., vol. 24, Amer. Math. Soc., Providence, R. I., 1973, pp. 213-220.

DEP ARTMENT OF MATHEMATICS, UNIVERSITY OF SOUTH CAROLINA, COLUMBIA, SOUTH CAROLINA 29208 